Research Paper

\title{
Classifying Non-Small Cell Lung Cancer by Status of Programmed Cell Death Ligand 1 and Tumor-Infiltrating Lymphocytes on Tumor Cells
}

\author{
Shaohua Cui, Lili Dong, Jialin Qian, Lin Ye, Liyan Jiang ${ }^{\circledR}$ \\ Department of Respiratory Medicine, Shanghai Chest Hospital, Shanghai Jiao Tong University \\ $\triangle$ Corresponding author: Liyan Jiang, Department of Respiratory Medicine, Shanghai Chest Hospital, Shanghai Jiao Tong University, 241\# HuaiHai (W.) Rd, \\ Shanghai, 200030, China. Tel: +86 13916146759 Fax: +86 021-32260856 E-mail: Jiang_liyan2000@126.com \\ (C) Ivyspring International Publisher. This is an open access article distributed under the terms of the Creative Commons Attribution (CC BY-NC) license \\ (https://creativecommons.org/licenses/by-nc/4.0/). See http://ivyspring.com/terms for full terms and conditions.
}

Received: 2017.07.10; Accepted: 2017.10.06; Published: 2018.01.01

\begin{abstract}
Purpose: To explore the possible correlation between programmed death ligand 1 (PD-LI)/tumor-infiltrating lymphocytes (TIL) status and clinical factors in non-small cell lung (NSCLC).

Materials and Methods: A total of 126 surgical NSCLC samples with stage I to IIIA were retrospectively collected and analyzed. Immunohistochemistry $(\mathrm{IHC})$ assays were used to detect PD-LI protein expression. PD-LI positivity on tumor cells was defined by positive tumor cell (TC) percentage using $5 \%$ cutoff value.

Results: Thirty-seven patients $(29.4 \%)$, thirty patients $(23.8 \%)$, six patients $(4.8 \%)$ and fifty-three patients (42\%) were classified as type I (PD-LI+, TIL+), type II (PD-LI-, TIL-), type III (PD-LI+, TIL-) and type IV (PD-LI-, TIL+) tumor environments according to PD-LI/TIL status, respectively. Statistical differences could be observed in factors including gender $(P<0.001)$, smoking status $(P<0.001)$, age $(P=0.002)$, histological types $(P<0.001)$, EGFR mutation $(P=0.008)$ and KRAS mutation $(P=0.003)$ across the four type tumors. Type I tumors were associated with ever smoking, non-adenocarcinoma histological types and KRAS mutation. Type II tumors were associated with female gender, never-smoking, adenocarcinoma histological types and EGFR mutation. Type III tumors were associated with ever smoking and type IV tumors were associated with female gender and EGFR mutation.

Conclusion: Clinical factors associated with NSCLC microenvironment types based on PD-L1/TIL differed a lot across different types. The findings of this study may help to facilitate the understanding of the relationship between tumor microenvironment and clinical factors, and also the selecting of patients for combination immunotherapies.
\end{abstract}

Key words: Programmed death ligand 1 (PD-L1); tumor-infiltrating lymphocytes (TIL); non-small cell lung cancer (NSCLC); immunotherapy.

\section{Introduction}

Avoiding immune destruction is one of the hallmarks of cancer recognized in recent years which indicates that immune system plays an important role in tumor growth and progression [1]. Programmed death ligand 1 (PD-L1, also known as B7-H1 or $\mathrm{CD} 274)$ plays an integral role with its receptor programmed death-1 receptor (PD-1) in attenuating T-cell activation resulting in the downregulation of immune response against tumor [2, 3]. Recently, PD-1/PD-L1 have been identified as targets of cancer immunotherapy and immunocheckpoint inhibitors targeting PD-1/PD-L1 pathway have been developed as important anticancer therapies in several cancer types, including non-small-cell lung cancer (NSCLC) [4-10]. To date, most studies revealed that high PD-L1 expression levels in tumor cells correlated with an increased response to PD-L1 inhibitors in NSCLC [7, 9 , 11, 12]. However, the cut-off values used for defining PD-L1 positivity varied from $1 \%$ to $50 \%$ in these studies based on the antibodies used for PD-L1 
staining. It is still urgent to select patients who will be benefit from anti-PD-L1 therapy currently.

PD-L1 expression in tumor cells (TC) has been reported to be significantly associated with tumor-infiltrating lymphocytes (TIL) [13], suggesting that tumor microenvironment played an important role in tumor growth and progression. In an effort to predict patients who will respond to PD-1/PD-L1 blockade, it has already been proposed that tumors can be classified into four groups on the basis of their PD-L1 status and presence or absence of TIL [14-16]. These four groups are: type I - PD-L1 positive with TILs driving adaptive immune resistance, type II PD-L1 negative with no TIL indicating immune ignorance, type III - PD-L1 positive with no TIL indicating intrinsic induction and type IV - PD-L1 negative with TIL indicating the role of other suppressors in promoting immune tolerance. Tailoring cancer immunotherapy according to the tumor microenvironment types may be a promising field of cancer treatment. Currently, evidence was mature mainly in melanoma and however, in NSCLC the evidence is insufficient. Thus, in this study we retrospectively collected NSCLC samples at our institute to explore possible correlation between PD-L1/TIL status and clinical factors.

\section{Materials and Methods}

\section{Tissue samples and collection of clinical information}

A total of 126 primary NSCLC surgical samples with stage I to IIIA were retrospectively collected in the present study. These samples were derived from patients who underwent surgical procedures from September 2009 to August 2012 in Shanghai Chest Hospital, Shanghai Jiao Tong University. Formalin fixed and paraffin embedded (FFPE) sections of all samples were stained with hematoxylin and eosin (HE) and confirmed the NSCLC diagnosis.

Common clinical factors including age at the time of diagnosis, gender, smoking status, histological type, tumor grade, clinical stage were retrospectively collected. PD-L1 protein expression, TIL status, EGFR mutation and KRAS mutation were detected in all samples. The detailed detection methods for these molecular were described in the following part.

This study was approved by the Ethics Committees of Shanghai Chest Hospital, Shanghai Jiao Tong University. Written informed consent was obtained from all patients.

\section{Molecular detection}

PD-L1

Immunohistochemistry (IHC) assays were used to detect PD-L1 protein expression in NSCLC samples. Placenta and MDA-MB-231 were used as positive control for PD-L1 IHC, and MCF-7 was used as negative control for PD-L1 IHC. A rabbit anti-human PD-L1 (E1L3N) monoclonal antibody (1:300, CST\#13684, Cell Signaling Technology) was used for PD-L1 IHC staining. The IHC procedure is briefly described as follows. Deparaffinized and rehydrated FFPE sections were immersed in high $\mathrm{pH}$ target retrieval solution in a pressure cooker (PTlink module, DAKO) at $97^{\circ} \mathrm{C}$ for 35 minutes. Sections were then treated with $2.5 \% \mathrm{H}_{2} \mathrm{O}_{2}$ in methanol for 15 minutes, followed by an incubation of protein block solution (PBS with $2 \%$ cold water fish skin gelatin, 1\% casein, $2 \%$ normal goat serum and $0.1 \%$ Tween-20) for 30 minutes. Subsequently, sections were incubated with primary antibody at room temperature for 60 minutes. After washing twice with TBS-T, sections were incubated with the EnVision+ -HRP labeled secondary antibody (K4003, DAKO) for 30 minutes. After a further two washes in TBS-T, slides were finally visualized using $\mathrm{DAB}$ substrate-chromagen (K3468, DAKO). PD-L1 expression was evaluated on tumor cells. PD-L1 positivity on tumor cells was defined by positive TC percentage using 5\% cutoff value.

\section{EGFR and KRAS}

EGFR and KRAS mutations were detected by amplification refractory mutation system (ARMS) method. Human EGFR Gene 29 Mutations Fluorescence Polymerase Chain Reaction (PCR) Diagnostic Kit and Human KRAS Gene 7 Mutations Fluorescence Polymerase Chain Reaction (PCR) Diagnostic Kits (Amoy Diagnostics, Xiamen, China) were used for the EGFR and KRAS mutation detections in this study. Prior to ARMS procedures, DNA was isolated from frozen tissues using the Puregene DNA extraction kit (Qiagen, Maryland, USA) according to the manufacturer's instructions. For detection of EGFR and KRAS mutations, the concentration of each DNA sample was normalized to $0.4 \mathrm{ng} / \mu \mathrm{L}$ and $0.66 \mathrm{ng} / \mu \mathrm{L}$, respectively.

\section{Statistical analysis}

The total samples were divided into four groups (type I, type II, type III and type IV) according to their PD-L1 expression status (positive or negative) at $5 \%$ cutoff and TIL status (with or without TIL). Type I, type II, type III and type IV samples represents PD-L1 positive with TIL (PD-L1+, TIL+), PD-L1 negative with no TIL (PD-L1-, TIL-), PD-L1 positive with no TIL (PD-L1+, TIL-) and PD-L1 negative with TIL (PD-L1-, TIL+), respectively. Proportions of clinical factors collected in this study was calculated in all 
four groups to explore the possible correlation between tumor environment and clinical factors. All enumeration data were presented as "count (percentage)". Fisher's exact test was used for comparing the differences of basic characteristics among four groups. Overall survival (OS) was calculated in all cohort and the four groups, respectively. The time-to-event OS was defined as the time from the date surgery was conducted until the date of death from any cause. Each of the median OS data was presented as a point estimation along with a 95\% confidence interval. Kaplan-Meier method was used to estimate OS, and log-rank tests (pairwise over strata) were used to analyze differences between subgroups. All confidence intervals were two-sided, and $P$ values less than 0.05 were considered statistically significant. All statistical analyses in this study were performed using SPSS software, version 13.0 (SPSS Inc., Chicago, IL, USA). The cutoff date of the study was April 16, 2017

\section{Results}

Demographic and clinicopathologic characteristics of the 126 patients have been shown in Table 1. The median age of all patients was 59 years (Range 32-78 years). These patients tended to be male gender $(72.2 \%)$, ever-smokers $(58.7 \%)$, and most patients (55.6\%) were staged IIIA. Twenty-four $(19.0 \%)$ patients and six $(4.8 \%)$ patients harbored EGFR mutation and KRAS mutation, respectively. Ninety $(71.4 \%)$ of the 126 samples were identified to concurrent TIL. Using $5 \%$ as the cut-off values for PD-L1 positivity, $43(34.1 \%)$ of the 126 samples showed PD-L1 membranous staining.
Thirty-seven patients $(29.4 \%)$, thirty patients $(23.8 \%)$, six patients $(4.8 \%)$ and fifty-three patients $(42 \%)$ were classified as type I (PD-L1+, TIL+), type II (PD-L1-, TIL-), type III (PD-L1+, TIL-) and type IV (PD-L1-, TIL+) tumor environments according to PD-L1/TIL status, respectively (Figure 1). The association between tumor environment and clinical factors was shown in Table 1. Among the four groups, statistical differences could be observed in factors including gender $(P<0.001)$, smoking status $(P<0.001)$, age $(P=0.002)$, histological types $(P<0.001)$, EGFR mutation $(P=0.008)$ and KRAS mutation $(P=0.003)$.

For type I tumors, patients tended to be ever smokers $\quad(89.2 \%), \quad \geq 60$ years $(75.7 \%)$, non-adenocarcinoma histological types $(81.1 \%)$. One sample $(2.7 \%)$ was identified to harbor EGFR mutation and five samples $(13.5 \%)$ were identified to harbor KRAS mutation. For type II tumors, patients tended to be female gender $(46.7 \%)$ compared with type I tumors, never-smokers (63.3\%), adenocarcinoma histological types (73.3\%). Eight samples $(26.7 \%)$ and one sample (3.3\%) harbored EGFR mutation and KRAS mutation, respectively. For type III tumors, four patients $(66.7 \%)$ were ever smokers and three samples $(50 \%)$ were adenocarcinoma histological type. One sample $(16.7 \%)$ was detected EGFR mutation. For type IV tumors, patients tended to be female gender (35.8\%) compared with type I tumors. Twenty-seven patients $(50.9 \%)$ and twenty-six patients $(49.1 \%)$ were never smokers and ever smokers, respectively. Fourteen samples $(26.4 \%)$ were identified to harbor EGFR mutations while none samples were detected to harbor KRAS mutations.

Table 1. Basic information of the 126 NSCLC patients and correlation between four tumor types according to PD-LI expression and TIL status and clinical parameters

\begin{tabular}{|c|c|c|c|c|c|c|c|}
\hline Characteristics & & $\begin{array}{l}\text { Total } \\
\mathrm{N}=126 \\
\mathrm{~N}(\%)\end{array}$ & $\begin{array}{l}\text { Type I } \\
\text { N=37, 29.4\% } \\
\text { n (\%) }\end{array}$ & $\begin{array}{l}\text { Type II } \\
\mathrm{N}=30,23.8 \% \\
\mathrm{n}(\%)\end{array}$ & $\begin{array}{l}\text { Type III } \\
\mathrm{N}=6,4.8 \% \\
\mathrm{n}(\%)\end{array}$ & $\begin{array}{l}\text { Type IV } \\
\mathrm{N}=53,42 \% \\
\mathrm{n}(\%)\end{array}$ & $\begin{array}{l}\text { P (Type I vs. } \\
\text { Type II vs. Type } \\
\text { III vs. Type IV) }\end{array}$ \\
\hline \multirow[t]{2}{*}{ Gender } & Male & $91(72.2)$ & $36(97.3)$ & $16(53.3)$ & $5(83.3)$ & $34(64.2)$ & $<0.001^{*}$ \\
\hline & Female & $35(27.8)$ & $1(2.7)$ & $14(46.7)$ & $1(16.7)$ & $19(35.8)$ & \\
\hline \multirow[t]{2}{*}{ Smoking } & Never smokers & $52(41.3)$ & $4(10.8)$ & $19(63.3)$ & $2(33.3)$ & $27(50.9)$ & $<0.001^{*}$ \\
\hline & Ever Smokers & $74(58.7)$ & $33(89.2)$ & $11(36.7)$ & $4(66.7)$ & $26(49.1)$ & \\
\hline \multirow[t]{2}{*}{ Age } & $<60$ years & $63(50.0)$ & $9(24.3)$ & $18(60.0)$ & $4(66.7)$ & $32(60.4)$ & $0.002^{*}$ \\
\hline & $\geq 60$ years & $63(50.0)$ & $28(75.7)$ & $12(40.0)$ & $2(33.3)$ & $21(39.6)$ & \\
\hline \multirow[t]{2}{*}{ Histological types } & $\mathrm{AD}$ & $63(50.0)$ & $7(18.9)$ & $22(73.3)$ & $3(50)$ & $31(58.5)$ & $<0.001^{*}$ \\
\hline & Non-AD & $63(50.0)$ & $30(81.1)$ & $8(26.7)$ & $3(50)$ & $22(41.5)$ & \\
\hline \multirow[t]{2}{*}{ Tumor grade } & Low differentiation & $33(26.2)$ & $13(35.1)$ & $6(20)$ & $1(16.7)$ & $13(24.5)$ & 0.522 \\
\hline & Other grades & $93(73.8)$ & $24(64.9)$ & $24(80)$ & $5(83.3)$ & $40(75.5)$ & \\
\hline \multirow[t]{2}{*}{ Clinical stage } & I-II & $56(44.4)$ & $17(45.9)$ & $16(53.3)$ & $3(50)$ & $20(37.7)$ & 0.551 \\
\hline & IIIA & $70(55.6)$ & $20(54.1)$ & $14(46.7)$ & $3(50)$ & $33(62.3)$ & \\
\hline \multirow[t]{2}{*}{ EGFR mutation } & + ve & $24(19.0)$ & $1(2.7)$ & $8(26.7)$ & $1(16.7)$ & $14(26.4)$ & $0.008^{*}$ \\
\hline & -ve & $102(81.0)$ & $36(97.3)$ & $22(73.3)$ & $5(83.3)$ & $39(73.6)$ & \\
\hline \multirow[t]{2}{*}{ KRAS mutation } & + ve & $6(4.8)$ & $5(13.5)$ & $1(3.3)$ & 0 & 0 & $0.003^{*}$ \\
\hline & -ve & $120(95.2)$ & $32(86.5)$ & $29(96.7)$ & $6(100)$ & $53(100)$ & \\
\hline
\end{tabular}

Abbreviations: AD: adenocarcinoma; EGFR: epidermal growth factor receptor; KRAS: Kirsten rat sarcoma viral oncogene; NSCLC: non-small cell lung cancer; TIL: tumor infiltrating lymphocytes.

+ve and -ve represent positive and negative results. ${ }^{*} P \leq 0.05$ 

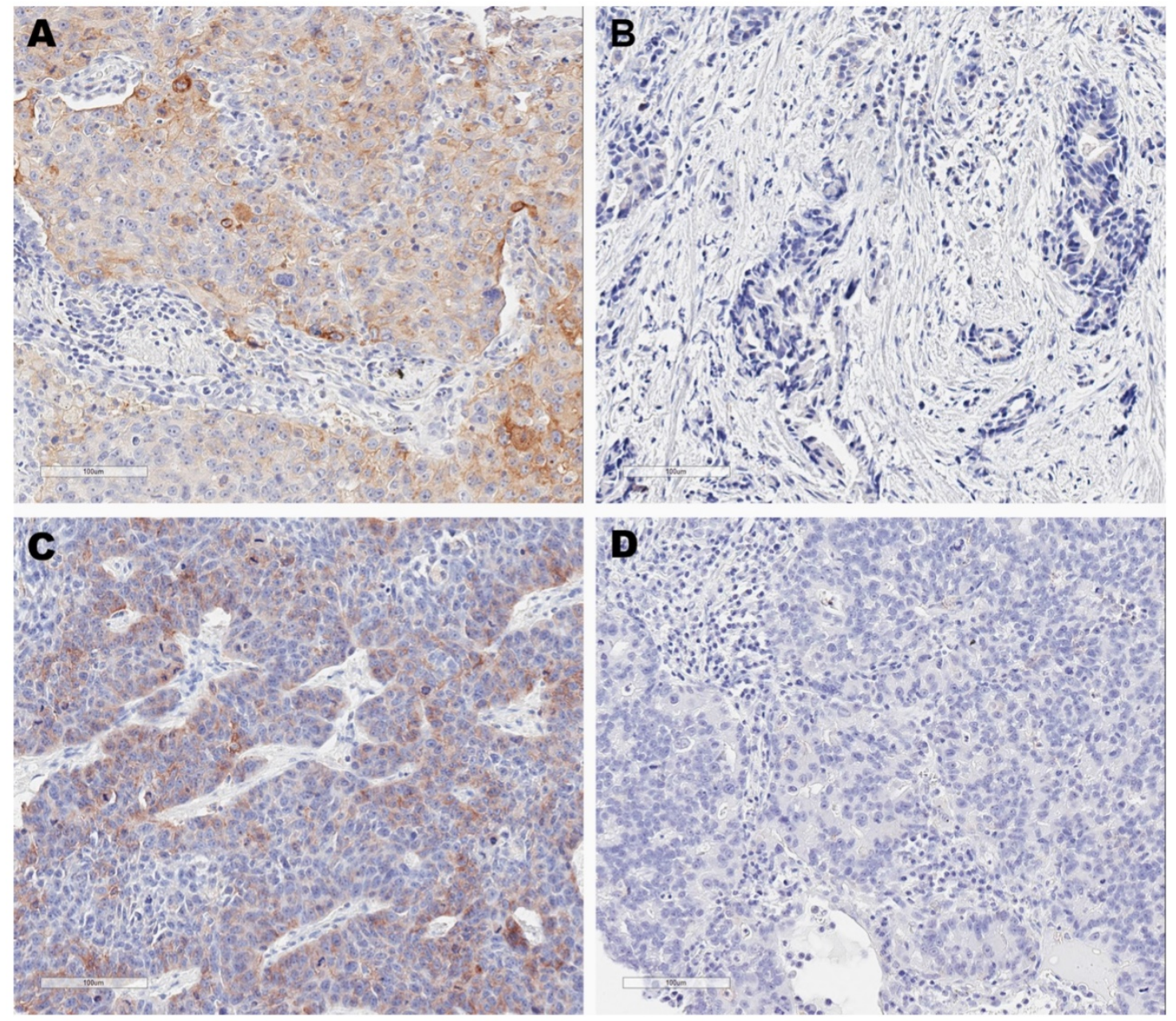

Figure 1. Immunohistochemistry images for four tumor microenvironment types based on programmed cell death ligand 1 (PD-LI) and tumor-infiltrating lymphocytes (TIL) status. These are representative images for type I tumors (PD-LI+, TIL+) (A), type II tumors (PD-L1-, TIL-) (B), type III tumors (PD-LI+, TIL-) (C) and type IV tumors (PD-LI-, TIL+) (D). PD-LI positivity was defined at $5 \%$ cut-off.

The median follow-up time for all cohort was 41.1 months. Eighty-five $(67.5 \%)$ of the 126 patients died at the cutoff date. The median OS for all cohort was 44.1 months (95\% CI: 33.9-70.0 months). The median OS for patients with type I (PD-L1+, TIL+), type II (PD-L1-, TIL-), type III (PD-L1+, TIL-) and type IV (PD-L1-, TIL+) tumors were 26.3 months $(95 \% \mathrm{CI}$ 11.1-41.5 months), 63.4 months (95\%CI 27.5-99.2 months), 40.3 months (95\% CI not applicable), and 45.4 months (95\%CI 34.5-56.2 months), respectively (Figure 2).

\section{Discussion}

In this study, we demonstrated the correlation between tumor types based on PD-L1/TIL status and clinical factors in 126 surgical samples. Our results revealed that type I (PD-L1+, TIL+) tumors were associated with ever smoking, non-adenocarcinoma histological types and KRAS mutation; Type II (PD-L1-, TIL-) tumors were associated with female gender, never-smoking, adenocarcinoma histological types and EGFR mutation; Type III (PD-L1+, TIL-) tumors were relatively rare compared with other types and were associated with ever smoking; and type IV (PD-L1-, TIL+) tumors, the most common types, were associated with female gender and EGFR mutation.

We identified that about $30 \%$ NSCLC were classified as type I (PD-L1+, TIL+) tumors. It has been reported that patients with type I tumor environments are largely responding to checkpoint inhibitions [14, 17]. Patients with type I tumors are most likely to benefit from single-agent anti-PD-1/PD-L1 inhibition as these tumors have evidence of preexisting intra-tumor $\mathrm{T}$ cells that are turned off by PD-L1 engagement [15]. In this study, we found a correlation between type I tumors and clinical factors including ever smoking, non-adenocarcinoma histological types, suggesting that patients with ever-smoking history and non-adenocarcinoma subtypes may be 
selected to receive single-agent anti-PD-1/PD-L1 therapy. Besides, KRAS targeted therapies may be considered as $13.5 \%$ of these patients were identified to harbor KRAS mutation according to our results. The OS was relatively short than other groups in our study and the reason remains unknown. The relatively high prevalence of KRAS mutation in type I tumor group may contribute in part to the poor prognosis of this group regardless of other reasons [18].

23.8\% NSCLC samples were divided into type II (PD-L1-, TIL-) tumors. For immunotherapy, patients with type II tumors would not consider to receive single-agent checkpoint blockade given the lack of preexisting T-cell infiltrates. Instead, combination therapy that can bring $\mathrm{T}$ cells into tumors and then avoid them being turned off, such as the combination of anti- cytotoxic $\mathrm{T}$ lymphocyte-associated antigen- 4 (CTLA-4) and anti-PD-1, would be considered in this scenario [15]. In addition, type II tumors were associated with female gender, never-smoking, adenocarcinoma histological types and EGFR mutation in our study. These factors are also regarded as the factors that may potentially predict better response to epidermal growth factor receptor tyrosine kinase inhibitors (EGFR-TKIs) therapies. Thus, it is more likely for patients with type II tumors to harbor EGFR mutations and have the chance to receive EGFR-TKIs therapies. The OS of patients with type II tumors was longer than other groups which conflicted with the theory that these patients were predicted to have very poor prognosis since the lack of detectable immune reactions. We reckoned that postsurgical therapies may contribute to the confusing results and further studies are needed to confirm the findings.

Only about 5\% NSCLC samples were classified as type III (PD-L1+, TIL-) tumors in this study. This type may happen when PD-L1 is constitutively expressed on tumor cells through oncogenic signaling pathways. Without TIL in the tumor, PD-1/PD-L1 blockade will unlikely lead to a T-cell response to tumors, highlighting that PD-L1 positivity alone cannot be taken as a predictive factor for response to anti-PD-1/PD-L1 therapies [15]. Combination therapies which can bring $T$ cells into tumors and then avoid them being turned off, such as the combination of anti-CTLA-4 and anti-PD-1, would also be considered in patients with type III tumors. We only found that ever-smoking history may correlate with this type in this study.

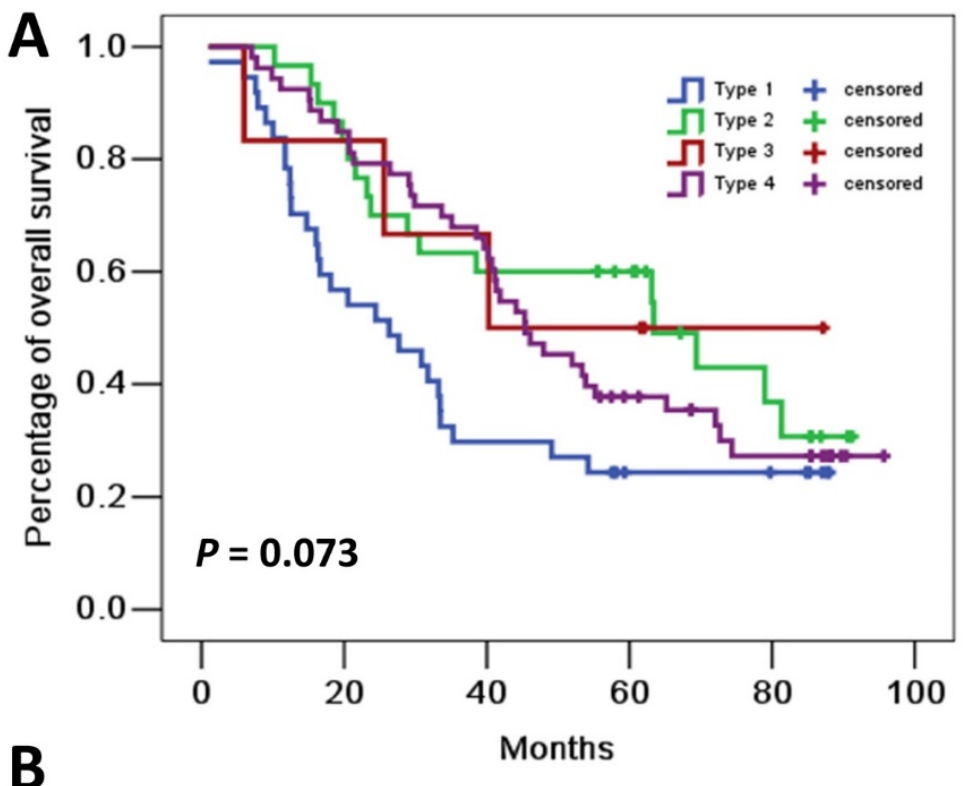

\begin{tabular}{|l|c|c|c|c|}
\hline Type & I & II & III & IV \\
\hline I & I & $0.029^{*}$ & 0.234 & $0.050^{*}$ \\
\hline II & $0.029^{*}$ & I & 0.972 & 0.395 \\
\hline III & 0.234 & 0.972 & I & 0.656 \\
\hline IV & $0.050^{*}$ & 0.395 & 0.656 & I \\
\hline
\end{tabular}

Figure 2. Overall survival curves for four tumor microenvironment types based on programmed cell death ligand 1 (PD-L1) and tumor-infiltrating lymphocytes (TIL) status. (A) Kaplan-Meier curves for the four tumor microenvironment types. Tick marks represent censored data. (B) $P$ values of pairwise over strata comparison between different groups. $* P \leq 0.05$. 
Type IV (PD-L1-, TIL+) tumors accounted for the largest proportion (more than $40 \%$ ) of all tumor environment types according to our results. Patients with type IV tumors containing TIL, however, no obvious adaptive resistance existed. These patients will be considered other non-PD-1/PD-L1 checkpoint receptors or immunosuppressive pathways and non-T-cell effector strategies [15]. 26.4\% samples of type IV tumors were demonstrated to harbor EGFR mutations and may consider EGFR-TKIs in this scenario.

The limitations of this study should be clarified. First of all, this is a single-center retrospective study with a relative small sample size. Postsurgical therapies were not considered because of the retrospective design and these treatment situations may affect the OS data. Secondly, more patients with male gender and stage IIIA were included in the study. Thirdly, the antibody used for PD-L1 staining in this study was not consistent with previously studies. The anti-PD-L1 antibody E1L3N was used for IHC analysis in our study. Lastly, this is a descriptive study and the results should be confirmed by further studies with comparisons and robust sample size.

To the best of our knowledge, this is the earliest study conducted in NSCLC samples to explore clinical factors associated with PD-L1/TIL status. The simple stratification of NSCLC into four types based on their PD-L1/TIL sets a framework to identify which pathways should be targeted to elicit the best response. The findings of this study may help to facilitate the understanding of the relationship between tumor microenvironment and clinical factors, and also the selecting of patients for combination immunotherapies.

\section{Acknowledgments}

This project was supported by the National Nature Science Foundation of China (Grant No. 81472175), Medical Guidance Project of Shanghai Science and Technology Committee (Grant No. 15411961500) and Policy Research Project of Shanghai Health and Family Planning Commission (Grant No. 2017HP18).

\section{Competing Interests}

The authors have declared that no competing interest exists.

\section{References}

1. Hanahan D, Weinberg RA. Hallmarks of cancer: the next generation. Cell. 2011; 144: 646-74.

2. Pardoll DM. The blockade of immune checkpoints in cancer immunotherapy. Nat Rev Cancer. 2012; 12: 252-64.

3. Gatalica Z, Snyder C, Maney T, et al. Programmed cell death 1 (PD-1) and its ligand (PD-L1) in common cancers and their correlation with molecular cancer type. Cancer Epidemiol Biomarkers Prev. 2014; 23: 2965-70.
4. Topalian SL, Hodi FS, Brahmer JR, et al. Safety, activity, and immune correlates of anti-PD-1 antibody in cancer. N Engl J Med. 2012; 366: 2443-54.

5. Brahmer JR, Tykodi SS, Chow LQ, et al. Safety and activity of anti-PD-L1 antibody in patients with advanced cancer. N Engl J Med. 2012; 366: 2455-65.

6. Herbst RS, Baas P, Kim DW, et al. Pembrolizumab versus docetaxel for previously treated, PD-L1-positive, advanced non-small-cell lung cancer (KEYNOTE-010): a randomised controlled trial. Lancet. 2016; 387: 1540-50.

7. Borghaei H, Paz-Ares L, Horn L, et al. Nivolumab versus Docetaxel in Advanced Nonsquamous Non-Small-Cell Lung Cancer. N Engl J Med. 2015; 373: 1627-39.

8. Brahmer J, Reckamp KL, Baas P, et al. Nivolumab versus Docetaxel in Advanced Squamous-Cell Non-Small-Cell Lung Cancer. N Engl J Med. 2015; 373: 123-35.

9. Fehrenbacher L, Spira A, Ballinger M, et al. Atezolizumab versus docetaxel for patients with previously treated non-small-cell lung cancer (POPLAR): a multicentre, open-label, phase 2 randomised controlled trial. Lancet. 2016; 387: 1837-46.

10. Rittmeyer A, Barlesi F, Waterkamp D, et al. Atezolizumab versus docetaxel in patients with previously treated non-small-cell lung cancer (OAK): a phase 3, open-label, multicentre randomised controlled trial. Lancet. 2017; 389: 255-65.

11. Gettinger S, Rizvi NA, Chow LQ, et al. Nivolumab monotherapy for first-line treatment of advanced non-small-cell lung cancer. J Clin Oncol. 2016; 34: 2980-7.

12. Garon EB, Rizvi NA, Hui $\mathrm{R}$, et al. Pembrolizumab for the treatment of non-small-cell lung cancer. N Engl J Med. 2015; 372: 2018-28.

13. Velcheti V, Schalper KA, Carvajal DE, et al. Programmed death ligand-1 expression in non-small cell lung cancer. Lab Invest. 2014; 94: 107-16.

14. Taube JM, Anders RA, Young GD, et al. Colocalization of inflammatory response with B7-h1 expression in human melanocytic lesions supports an adaptive resistance mechanism of immune escape. Sci Transl Med. 2012; 4: $127 \mathrm{ra} 37$.

15. Teng MW, Ngiow SF, Ribas A, et al. Classifying cancers based on T-cell infiltration and PD-L1. Cancer Res. 2015; 75: 2139-45.

16. Sznol M, Chen L. Antagonist antibodies to PD-1 and B7-H1 (PD-L1) in the treatment of advanced human cancer. Clin Cancer Res. 2013; 19: 1021-34.

17. Taube JM, Klein A, Brahmer JR, et al. Association of PD-1, PD-1 ligands, and other features of the tumor immune microenvironment with response to anti-PD-1 therapy. Clin Cancer Res. 2014; 20: 5064-74.

18. Slebos RJ, Kibbelaar RE, Dalesio O, et al. K-ras oncogene activation as a prognostic marker in adenocarcinoma of the lung. N Engl J Med. 1990; 323: $561-5$. 